\title{
On the Design of PS-RCPT Codes for LTE System
}

\author{
Xiaofeng Long ${ }^{\dagger}$, Qingchun Chen ${ }^{\dagger}$, Pei Xiao ${ }^{\ddagger}$, Jinsong $\mathrm{Wu}^{*}$ \\ ${ }^{\dagger}$ Southwest Jiaotong University, Chengdu, 610031, China \\ ${ }^{\ddagger}$ University of Surrey, Guildford, Surrey, GU2 7XH, United Kingdom \\ *Bell Laboratories, Shanghai 201206, China \\ xiaofeng860530@163.com, qcchen@home.swjtu.edu.cn, p.xiao@surrey.ac.uk,wujs@ieee.org.
}

\begin{abstract}
The optimized weight spectrum sequence (OWSS) is utilized as a design criterion in this paper to determine the periodic puncturing pattern and the non-periodic puncturing pattern of partially systematic RCPT (PS-RCPT) codes for LTE systems. It is shown that the OWSS-criterion based PS-RCPT codes outperform the pseudo-random puncturing (PRP) based PS-RCPT codes. Meanwhile, it is unveiled that, the puncturing ratio of information bits should be carefully determined in PSRCPT codes generation to achieve reasonable tradeoff between the waterfall region and the error floor region performance.
\end{abstract}

Index Terms-Optimized weight spectrum sequence, partially systematic Turbo codes, puncturing pattern design , LTE.

\section{INTRODUCTION}

Turbo codes have attracted considerable attention due to their impressive performance to approach Shannon limits. Rate-compatible puncturing provides an effective method to realize adaptive error control coding. Rate compatible puncturing Turbo (RCPT) codes are needed in different systems to enable adaptive modulation and coding schemes (MCS) as well as the hybrid ARQ schemes. Therefore, the RCPT code design plays an important role in technical standards, such as long term evolution (LTE)[9]. In addition to the rate-compatibility restriction, the choice of design criteria is another important factor which has an impact on the RCPT code design [2]-[4]. In the early stage of RCPT code design, uniform interleavers are generally assumed to avoid the tedious analysis owing to the permutation by internal interleaver. However, the performance of the devised RCPT code based on such an assumption cannot always be guaranteed, especially when some practically devised internal interleaver is utilized. In order to facilitate the design of RCPT codes for a given interleaver, which would always be specified in the technical standards, Rosnes proposed a two-step procedure in [5] to determine the optimal periodic puncturing pattern based on free distance of the Turbo code, which can be calculated by using the constrained subcode algorithm in [7]. As expected, better RCPT codes can be obtained [5]. However, it must be addressed that, all these previous works only considered the systematic RCPT (S-RCPT) code design (namely, no systematic bits are allowed to be punctured). Recently, it has been foound that, by allowing some systematic bits to be perforated, the partially systematic RCPT (PS-RCPT) codes

This work was supported by the National Basic Research Program of China (973 Program No. 2012CB316100), the NSFC under grant No.60872013/61032002 and the Young Innovative Research Team of Sichuan Province under grant No.2011JTD0007. with code rate $1 / 2$ can achieve even better reliability than the $1 / 3$ code rate mother codes, and the pseudo-random puncturing (PRP) scheme was proposed to devise the 1/2-rate PRP-PSRCPT codes in [8].

Motivated by the advancement in the PS-RCPT code design, in this paper we focus on the improved periodic puncturing pattern design and the improved non-periodic puncturing pattern design for PS-RCPT codes with different code rates. More specifically, instead of the pseudo-random puncturing design, we aim to improve design criterion to generate PS-RCPT codes with different code rates. Our previous analysis has already unveiled that, the optimized weight spectrum sequence (OWSS) criterion is a good choice to devise S-RCPT codes with guaranteed performance [6]. In this paper, we apply the OWSS criterion to design PS-RCPT codes. Our analysis shows that, the OWSS-criterion based PS-RCPT codes outperform the pseudo-random puncturing (PRP) based PS-RCPT codes. Moreover, it is shown that the puncturing ratio of information bits should be carefully determined in PS-RCPT codes generation to achieve reasonable tradeoff between the waterfall region and the error floor region performance. Meanwhile, unless otherwise stated, all Turbo codes discussed in the rest of this paper are the standard Turbo code specified by LTE technical specifications [9]. Hence, all PS-RCPT codes and the related design algorithms are applicable in LTE systems. On the other hand, the similar analysis can be readily extended to the PS-RCPT code design for other Turbo codes.

The remainder of this paper is organized as follows. In Section II, the optimized puncturing pattern design criteria are reviewed. The OWSS-criterion based periodic puncturing pattern design and non-periodic puncturing pattern design for PS-RCPT codes are presented in Section III. Simulation results are presented in Section IV to validate the performance of the OWSS-criterion based RCPT codes. Finally, some discussions are presented in Section $\mathrm{V}$ to conclude this paper.

\section{RCPT CODE DESIGN CRITERIA}

Generally speaking, the design of RCPT codes requires an effective method to evaluate the possible performance loss due to the removal of some code symbols, so that we may decide whether or not to keep the puncturing pattern candidate. Intuitively, simulations can be performed to examine every possible candidate puncturing pattern so as to determine the optimal one. However, this would become prohibitively complicated, especially when there are too many candidate 


$$
Z_{i}^{L}=\left\{\left(-d_{\text {free }}^{i}, w_{d_{\text {free }}}^{i}, N_{d_{\text {free }}}^{i}\right),\left(-\left(d_{\text {free }}^{i}+1\right), w_{d_{\text {free }}+1}^{i}, N_{d_{\text {free }}+1}^{i}\right), \ldots,\left(-\left(d_{\text {free }}^{i}+L\right), w_{d_{\text {free }}+L}^{i}, N_{d_{\text {free }}+L}^{i}\right)\right\}(1)
$$

puncturing patterns to choose from. Unlike the computer simulation, performance estimate is more straightforward to assess or approximate the resultant performance for the given puncturing pattern with much less complexity. Basically, the differences among different design criteria are just the choices of different performance estimate method. Although there is no known performance model to characterize the whole performance behavior of Turbo codes, many research results have shown that, the input-output weight enumerating function (IOWEF) can be utilized to characterize the performance of Turbo codes within error floor region, to this end, the weight spectrum related criteria, such as the free-distance criterion, the minimum slope criterion, and the optimized weight spectrum sequence (OWSS) criterion, were proposed for designing the RCPT codes [2]-[6]. Our recent analysis shows that the error floor performance within moderate to high SNR region can be satisfactorily characterized by the first several low weight distance spectrum terms [10], which suggests that, the calculation overhead in the weight spectrum related criteria can be further reduced.

Let $d_{w}$ and $N_{w}$ denote the minimum Hamming weight of code words generated by input sequence with Hamming weight $w$ and the multiplicity of code words with weight $d_{w}$. Then the weight spectrum is denoted as the set of $\left(d_{w}, N_{w}\right)$ for $w=1, \ldots, w_{\max }$. The free-distance criterion requires that we select the candidate puncturing pattern yielding the largest free distance $d_{\text {free }}=\min \left\{d_{w}\right\}, w=1, \ldots, w_{\text {max }}$. The minimum slope criterion prefers to select the candidate puncturing pattern yielding the minimum slope (i.e., the minimum growth) in the first several low weight terms of the sequence of $\left(d_{w}, N_{w}\right)$. In the OWSS criterion, the candidate puncturing pattern which sequentially maximizes $d_{w}$ at first and minimizes $N_{w}$ thereafter will be selected. Compared with the free distance criterion, the OWSS criterion takes into consideration more weight spectrum terms which dominate the performance. Our previous analysis in [6] has validated that the OWSS criterion based S-RCPT codes can achieve better reliability for a given puncturing period. In fact, our recent analysis in [10] has already revealed that, in some cases, it is not the free distance term but the term with large enough multiplicity which dominates the error floor performance. This explicates why the OWSS criterion S-RCPT code outperforms the free distance criterion S-RCPT code. As we have addressed, the use of the OWSS criterion in place of the free distance criterion will not introduce too much additional calculation complexity. In this paper, we will generalize the OWSS criterion to determine the periodic puncturing patterns and non-periodic puncturing patterns for PS-RCPT codes.

\section{PS-RCPT CODE DESIGN}

In this section, we present at first a specific implementation metric to realize the OWSS criterion. The first $L$ terms of the weight spectrum of the $i$-th candidate puncturing pattern can be denoted by the following tri-tuple set in (1), where $w_{d}$ denotes the Hamming weight sum of all input sequences which lead to the code words with Hamming weight $d$ [6]. Here the distance term in the tri-tuple is artificially set to be a negative value in order to simplify the implementation of the OWSS criterion. It is known that, the OWSS criterion requires to maximize $d_{w}$ at first, and then to minimize $N_{w}$ in the second step. By using the negative distance terms in the weight spectrum sequence, the OWSS criterion could be transformed into the minimization of the following metric

$$
Z_{m}^{L}=\underset{\left\{0 \leq i \leq M_{m}-1\right\}}{\arg \min } Z_{i}^{L}
$$

where $m$ represents the puncturing level, $M_{m}$ is the number of puncturing pattern candidates considered in the $m$ th puncturing level. It must be addressed that, unlike the OWSS criterion in [4], where $\left\{\left(d_{\text {free }}^{i}, N_{d_{\text {free }}}^{i}\right),\left(\left(d_{\text {free }}^{i}+\right.\right.\right.$ $\left.\left.1), N_{d_{\text {free }}+1}^{i}\right), \ldots,\left(\left(d_{\text {free }}^{i}+L\right), N_{d_{\text {free }}+L}^{i}\right)\right\}$ is utilized, the metric in (2) accumulates input weight $w_{d}$ generating the same weight $d$. The advantage of the modified OWSS criterion is that, the BER performance is also taken into consideration in the optimal puncturing pattern design. More specifically, applying the metric in (2) leads to the selection of the optimal puncturing pattern in the following order: first $-d$ is minimized (i.e., $d$ is maximized), then $w_{d}$ is minimized, and finally $N_{d}$ is minimized.

We use the LTE standard Turbo codes [9] as an example to demonstrate the OWSS-criterion based RCPT design. Although these algorithms are designed for LTE standard Turbo codes, they can be readily extended to design other Turbo codes. It should be noted that those zero terminating bits for LTE standard Turbo codes will not be included in the puncturing process. In addition, there are two types of puncturing implementation, namely, the non-periodic puncturing and the periodic puncturing. Generally speaking, the non-periodic puncturing is better than the periodic puncturing. Our previous analysis reveals that the periodic puncturing converges to the non-periodic puncturing given that the puncturing period is sufficiently large [6].

\section{A. Periodic PS-RCPT Code Design}

A periodic or regular puncturing pattern for LTE standard rate $1 / 3$ Turbo Code can be specified by a binary puncturing matrix, where $P$ denotes the puncturing period. An example puncturing matrix $(P=8)$ is formed as follows:

$$
\left(\begin{array}{llllllll}
1 & 1 & 0 & 1 & 1 & 0 & 1 & 1 \\
1 & 0 & 1 & 1 & 0 & 1 & 0 & 0 \\
0 & 1 & 0 & 0 & 1 & 0 & 1 & 1
\end{array}\right)
$$

where the first row corresponds to systematic bits sequence, the second row and the last row correspond to two component 
parity bit sequences, respectively. A matrix element being equal to 0 indicates that the corresponding symbol will be punctured, otherwise, it will be kept. Obviously, the resultant code by using the example puncturing table in (3) belongs to PS-RCPT codes since some systematic bits are also perforated. A greedy algorithm was proposed for the periodic puncturing design for S-RCPT codes in [5]. In this paper, we use a similar greedy algorithm to design the periodic puncturing pattern for PS-RCPT codes based on the OWSS criterion in (2). Moreover, it should be noted that the systematic bits are also included in the puncturing. In general, the puncturing

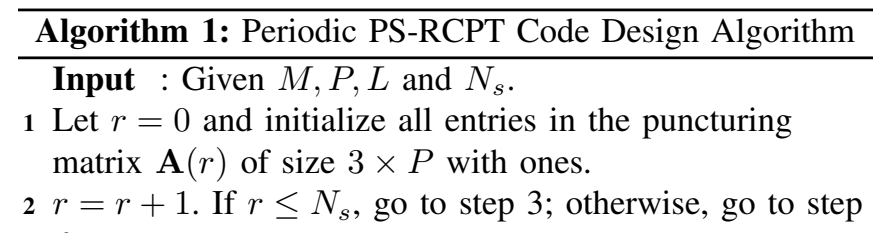
6.

3 Generate all possible candidates $\{\tilde{\mathbf{A}}(\mathbf{r})\}$ by setting one nonzero entry in the first row of $\mathbf{A}(r-1)$ as zero.

4 Compute the least $L$ terms in $Z$ for all candidates $\{\tilde{\mathbf{A}}(\mathbf{r})\}$.

5 Determine the optimal one from all candidates according to the OWSS criterion. Update $\mathbf{A}(r)$ with the selected candidate. Go to step 2.

6 Generate all possible candidates $\{\tilde{\mathbf{A}}(\mathbf{r})\}$ by setting one nonzero entry in the second and third rows of $\mathbf{A}(r-1)$ as zero.

7 Compute the least $L$ terms in $Z$ for all candidates $\{\tilde{\mathbf{A}}(\mathbf{r})\}$.

8 Determine the optimal one from all candidates according to the OWSS criterion. Update $\mathbf{A}(r)$ with the selected candidate. If $r>M$, terminate. Otherwise, go to step 2 . Output: $\mathbf{A}(r)$.

pattern design comprises of the following two steps: i). the first several terms of weight spectrum of all candidate puncturing patterns are computed; ii). the best puncturing positions are determined in accordance with the OWSS criterion. In order to avoid significant performance degradation in the decoding convergence for PS-RCPT codes, we assume the following constraints:

1. For the given punctured code rate and puncturing period $P$, set up a reasonable puncturing ratio of information bits.

2. During the successive puncturing, firstly determine the punctured position for the punctured information bits, then locate the optimal puncturing position for the parity-check symbols.

Our following analysis shows that, a larger puncturing ratio of information bits will lead to the PS-RCPT codes with larger free distance, which is desirable in order to achieve a lower error floor performance. However, if we remove too many information bits, the decoding convergence performance would deteriorate. Therefore, there exists a reasonable tradeoff between the achieved error floor performance and the decoding convergence in waterfall region, which can be transformed into a reasonable choice of puncturing ratio of information bits. Although there have been some results on the waterfall region performance modeling for Turbo codes [10], unfortunately, all the waterfall region performance estimates implicitly suggest that, there is a quite different relationship between the weight spectrum characteristics and the waterfall region decoding convergence performance. In this case, the weight spectrum criterion no longer works well to secure the decoding convergence of PS-RCPT codes. In order to guarantee the decoding convergence performance of the PS-RCPT codes, it is proposed to firstly determine the punctured position for the punctured information bits, then locate the optimal puncturing position for the parity-check symbols. Let the number of systematic bits to be punctured be denoted by $N_{s}\left(N_{s} / P\right.$ corresponds to the puncturing ratio of information bits), $M$ ( $N_{s} \leq M \leq 2 P$ ) denotes the total number of punctured symbols, the OWSS criterion based periodic puncturing pattern design procedure for PS-RCPT code can be depicted by Algorithm 1. The calculated weight spectrum of the optimized PS-RCPT codes for LTE standard Turbo code with information size 1248 are listed in Table 1 and Table 2, where $L=3$. The code rates of all RCPT codes in Table 1 are fixed as $1 / 2$, and influence of the systematic bit puncturing ratio $N_{s} / P$ (marked with "Ratio" in Table 1) is also presented. It can be seen that the OWSS criterion based PS-RCPT codes with sufficiently large information puncturing ratio (in this example, $N_{s} / P \geq 25 \%$ ) have larger free distance than the PRP-RCPT codes with systematic bit puncturing ratio $57.1 \%$ and $66 \%$ (only these two systematic bit puncturing ratios are included here since only they are reported in [8]), which implies that the optimized OWSS criterion based PS-RCPT codes can achieve a lower error floor. Meanwhile, it can be observed that a larger information puncturing ratio is desirable for the PS-RCPT codes to realize a lower error floor performance. For stance, when $N_{s} / P \geq 50 \%$, the PS-RCPT codes have a free distance up to 22 , which is very close to the free distance of its non-punctured mother Turbo code. However, the price to pay is the degradation in the decoding convergence performance within waterfall region, as will be verified by the numerical results in Section IV. Therefore, there exists a tradeoff when choosing the information puncturing ratio. In practical applications, we may determine the information puncturing ratio according to the application requirements. The weight spectrum of the OWSS-criterion based RCPT codes with different code rates are listed in Table 2, wherein the systematic bit puncturing ratio of the PS-RCPT codes is fixed as 50\%. Obviously, PS-RCPT codes and S-RCPT codes have different weight spectrum characteristics at different code rates. Compared with the S-RCPT codes, PS-RCPT ones have obviously larger free distance spectrum within both low and high code rate region.

\section{B. Non-Periodic PS-RCPT PS-RCPT Code Design}

In the previous design of S-RCPT codes, it is shown that the non-periodic puncturing S-RCPT codes may outperform the periodic puncturing ones [6]. The reason can be intuitively explicated by the additional constraint on the positions to be perforated by the periodic puncturing pattern constraint. In 


\begin{tabular}{|c|c|c|c|c|c|}
\hline \multirow{2}{*}{$\begin{array}{l}\text { Puncturing } \\
\text { category }\end{array}$} & \multirow{2}{*}{ Ratio } & \multirow{2}{*}{$\begin{array}{l}\text { Puncturing } \\
\text { pattern }\end{array}$} & \multicolumn{3}{|c|}{ Weight Spectrum } \\
\hline & & & $d$ & $N_{d}$ & $w_{d}$ \\
\hline \multirow{3}{*}{ Parent code } & \multirow{3}{*}{ - } & $\begin{array}{llllllll} & 1 & 1 & 1 & 1 & 1 & 1 & 1\end{array}$ & 24 & 1 & 2 \\
\hline & & 1111111111 & 25 & 1 & 3 \\
\hline & & 111111111 & 26 & 1 & 2 \\
\hline \multirow{3}{*}{ S-RCPT } & & 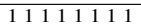 & 18 & 3 & 9 \\
\hline & & 0000011111 & 19 & 5 & 17 \\
\hline & & 00110110 & 20 & 6 & 23 \\
\hline \multirow{21}{*}{ PS-RCPT } & \multirow{3}{*}{$12.5 \%$} & $\begin{array}{lllllllllll} & 0 & 1 & 1 & 1 & 1 & 1 & 1 & 1\end{array}$ & 18 & 1 & 2 \\
\hline & & 0100011111 & 19 & 1 & 2 \\
\hline & & 000110101 & 20 & 4 & 13 \\
\hline & \multirow{3}{*}{$25 \%$} & $0 \begin{array}{lllllllll}0 & 1 & 0 & 1 & 1 & 1 & 1 & 1\end{array}$ & 20 & 3 & 9 \\
\hline & & 00100011111 & 21 & 5 & 18 \\
\hline & & 00011101111 & 22 & 3 & 10 \\
\hline & \multirow{3}{*}{$37.5 \%$} & 001000111111 & 21 & 3 & 9 \\
\hline & & 010111111 & 22 & 3 & 9 \\
\hline & & 00011010111 & 23 & 4 & 10 \\
\hline & \multirow{3}{*}{$50 \%$} & 01001101 & 22 & 5 & 14 \\
\hline & & 0101111111 & 24 & 3 & 9 \\
\hline & & 0.01111001111 & 25 & 5 & 19 \\
\hline & \multirow{3}{*}{$62.5 \%$} & 00100010001 & 22 & 1 & 2 \\
\hline & & 11011111 & 23 & 5 & 16 \\
\hline & & $\begin{array}{llllllllll}0 & 1 & 1 & 1 & 0 & 1 & 1 & 1\end{array}$ & 25 & 1 & 2 \\
\hline & \multirow{3}{*}{$75 \%$} & 01001000 & 23 & 3 & 7 \\
\hline & & 11011111 & 24 & 1 & 4 \\
\hline & & 01111111111 & 26 & 4 & 12 \\
\hline & \multirow{3}{*}{$87.5 \%$} & 00000010000 & 23 & 3 & 7 \\
\hline & & 11111111 & 25 & 1 & 4 \\
\hline & & 0111111111 & 26 & 1 & 3 \\
\hline \multirow{6}{*}{ PRP-RCPT } & \multirow{3}{*}{$57.1 \%$} & 1000110 & 16 & $\frac{1}{2}$ & 5 \\
\hline & & 01111001 & 20 & 3 & 8 \\
\hline & & 1111111 & 21 & 1 & 3 \\
\hline & \multirow{3}{*}{$66 \%$} & 100 & 18 & 2 & 5 \\
\hline & & 011 & 20 & 1 & 3 \\
\hline & & 111 & 22 & 3 & 7 \\
\hline
\end{tabular}

\begin{tabular}{|c|c|c|c|c|c|}
\hline \multirow{2}{*}{ Rate } & \multirow{2}{*}{$\begin{array}{c}\text { Puncturing } \\
\text { category }\end{array}$} & \multirow{2}{*}{$\begin{array}{c}\text { Puncturing } \\
\text { pattern }\end{array}$} & \multicolumn{3}{|c|}{ Weight Spectrum } \\
\hline & & & $d$ & $N_{d}$ & $w_{d}$ \\
\hline \multirow{3}{*}{$1 / 3$} & \multirow{3}{*}{ Parent code } & \multirow{3}{*}{-- } & 30 & 322 & 644 \\
\hline & & & 31 & 1 & 3 \\
\hline & & & 34 & 1 & 4 \\
\hline \multirow{6}{*}{$2 / 5$} & \multirow{3}{*}{ PS-RCPT } & 0.01000111001 & 24 & 1 & 2 \\
\hline & & 111111111 & 25 & 2 & 5 \\
\hline & & 11111111111 & 28 & 2 & 5 \\
\hline & \multirow{3}{*}{ S-RCPT } & $\begin{array}{llllllllll} & 1 & 1 & 1 & 1 & 1 & 1 & 1\end{array}$ & 22 & 1 & 2 \\
\hline & & 110011111111 & 23 & 3 & 8 \\
\hline & & 00111101110 & 24 & 3 & 9 \\
\hline \multirow{6}{*}{$1 / 2$} & \multirow{3}{*}{ PS-RCPT } & 00100011101 & 22 & 5 & 14 \\
\hline & & 00101111111 & 24 & 3 & 9 \\
\hline & & 0.0111101111 & 25 & 5 & 19 \\
\hline & \multirow{3}{*}{ S-RCPT } & 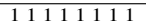 & 18 & 3 & 9 \\
\hline & & 00000011111 & 19 & 5 & 17 \\
\hline & & 0001110110 & 20 & 6 & 23 \\
\hline \multirow{6}{*}{$4 / 7$} & \multirow{3}{*}{ PS-RCPT } & 0010001101 & 19 & 2 & 5 \\
\hline & & 01010111101 & 20 & 4 & 13 \\
\hline & & 0001110111 & 21 & 8 & 30 \\
\hline & \multirow{3}{*}{ S-RCPT } & $\begin{array}{llllllllll} & 1 & 1 & 1 & 1 & 1 & 1 & 1\end{array}$ & 15 & 2 & 4 \\
\hline & & 0000011101 & 16 & 5 & 17 \\
\hline & & 001110100 & 17 & 11 & 36 \\
\hline \multirow{6}{*}{$2 / 3$} & \multirow{3}{*}{ PS-RCPT } & 00100011101 & 16 & 1 & 2 \\
\hline & & 0100011101 & 17 & 5 & 20 \\
\hline & & 00011101101 & 18 & 112 & 984 \\
\hline & \multirow{3}{*}{ S-RCPT } & 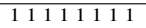 & 11 & 1 & 2 \\
\hline & & 00000101 & 12 & 5 & 14 \\
\hline & & 00100100 & 13 & 457 & 2424 \\
\hline \multirow{6}{*}{$4 / 5$} & \multirow{3}{*}{ PS-RCPT } & 0010001101 & 13 & 301 & 2236 \\
\hline & & 010001001 & 14 & 442 & 3525 \\
\hline & & 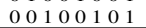 & 15 & 563 & 3998 \\
\hline & \multirow{3}{*}{ S-RCPT } & $\begin{array}{llllllllll} & 1 & 1 & 1 & 1 & 1 & 1 & 1\end{array}$ & 8 & 872 & 4508 \\
\hline & & 0000001000 & 9 & 2508 & 13246 \\
\hline & & 00100000 & 10 & 13437 & 83718 \\
\hline
\end{tabular}

this paper, we generalize the non-periodic puncturing pattern design in [6] to the PS-RCPT codes. It should be noted that, the greedy periodic puncturing design algorithm cannot be directly utilized since there are too many candidate puncturing patterns to choose from.

Let $N$ denote the information length, $N_{s}$ and $M$ denote the number of systematic bits to be punctured and the total number of symbols to be removed, respectively. The OWSS criterion based non-periodic puncturing pattern design procedure for PS-RCPT code can be described by Algorithm 2. Here it is worth mentioning that, we do not directly determine the optimal puncturing pattern according to the OWSS criterion at step 4 . Instead, we locate the position satisfying the OWSS criterion in an indirect manner. In fact, the puncturing positions should be determined in the following way as recommended

\begin{tabular}{|c|c|c|c|c|c|c|c|c|c|c|c|c|c|c|c|}
\hline & \multicolumn{15}{|c|}{ RCPT Code Rate } \\
\hline & \multicolumn{3}{|c|}{0.65} & \multicolumn{3}{|c|}{0.56} & \multicolumn{3}{|c|}{0.49} & \multicolumn{3}{|c|}{0.39} & \multicolumn{3}{|c|}{0.36} \\
\hline & $d_{w}$ & $N_{w}$ & $W$ & $d_{w}$ & $N_{w}$ & $w$ & $d_{w}$ & $N_{w}$ & $w$ & $d_{w}$ & $N_{w}$ & $w$ & $d_{w}$ & $N_{w}$ & $w$ \\
\hline \multirow{6}{*}{$\mathrm{P}$} & 10 & 68 & 335 & 12 & 1 & 2 & 16 & 3 & 7 & 20 & 3 & 6 & 20 & 1 & 2 \\
\hline & 11 & 4 & 11 & 14 & 73 & 629 & 17 & 3 & 7 & 21 & 1 & 1 & 21 & 3 & 5 \\
\hline & 12 & 532 & 3289 & 15 & 9 & 32 & 18 & 6 & 14 & 22 & 3 & 6 & 23 & 1 & 2 \\
\hline & 11 & 3 & 8 & 14 & 5 & 15 & 16 & 2 & 3 & 20 & 2 & 4 & 20 & 1 & 2 \\
\hline & 12 & 38 & 330 & 15 & 7 & 21 & 17 & 5 & 21 & 21 & 2 & 3 & 21 & 2 & 3 \\
\hline & 13 & 284 & 1847 & 16 & 20 & 62 & 18 & 11 & 32 & 23 & 5 & 9 & 22 & 1 & 2 \\
\hline \multirow{3}{*}{ NP } & 11 & 1 & 4 & 16 & 44 & 198 & 19 & 12 & 51 & 20 & 1 & 2 & 20 & 1 & 2 \\
\hline & 12 & 40 & 173 & 17 & 256 & 1279 & 20 & 102 & 490 & 21 & 1 & 1 & 21 & 1 & 1 \\
\hline & 13 & 185 & 922 & 18 & 400 & 2031 & 21 & 273 & 1359 & 22 & 9 & 34 & 22 & 2 & 4 \\
\hline
\end{tabular}

in [5]:

1. The puncturing position should be selected in such a way that, there will be the least increase in the first term of the OWSS criterion metric $Z$.

2. If there is a tie, the puncturing position should be selected in such a way that, there will be the least increase in the next term of the OWSS criterion metric $Z$.
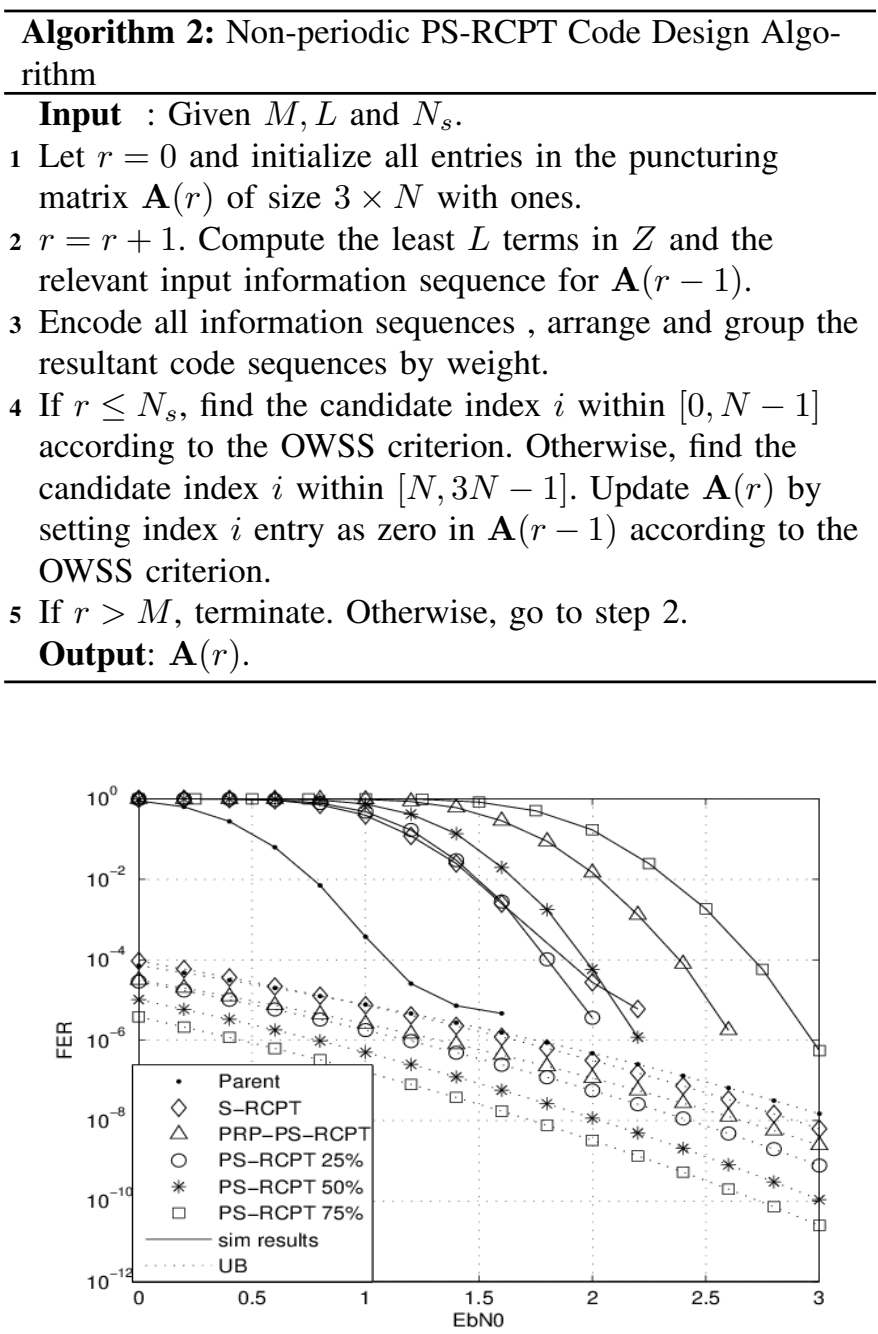

Fig. 1. FER of RCPT codes with code rate-1/2. $k=1248$. Solid line: simulation result, dot line: union bound estimation.

In order to demonstrate more clearly the optimal nonperiodic puncturing pattern, the spectrum sequence for the LTE standard Turbo code with $N=320$ and $N_{s}=80$ 


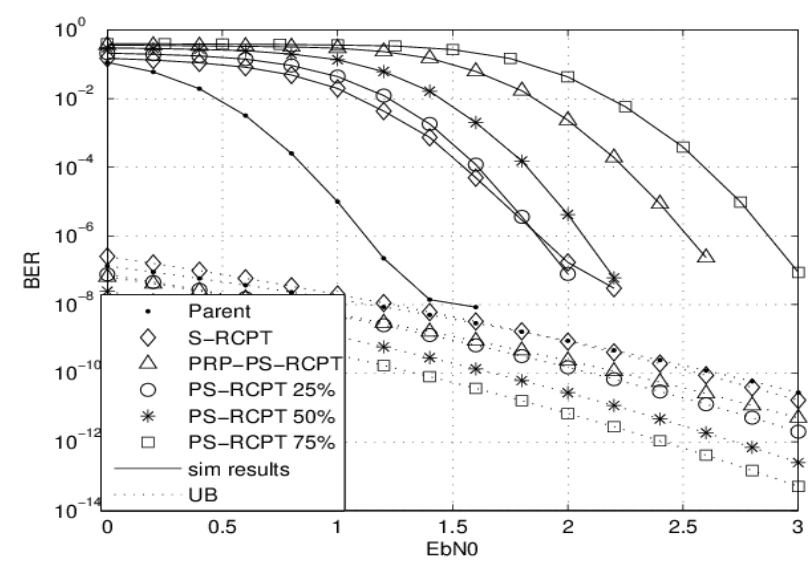

Fig. 2. BER of RCPT codes with code rate- $1 / 2 . k=1248$. Solid line: simulation result, dot line: union bound

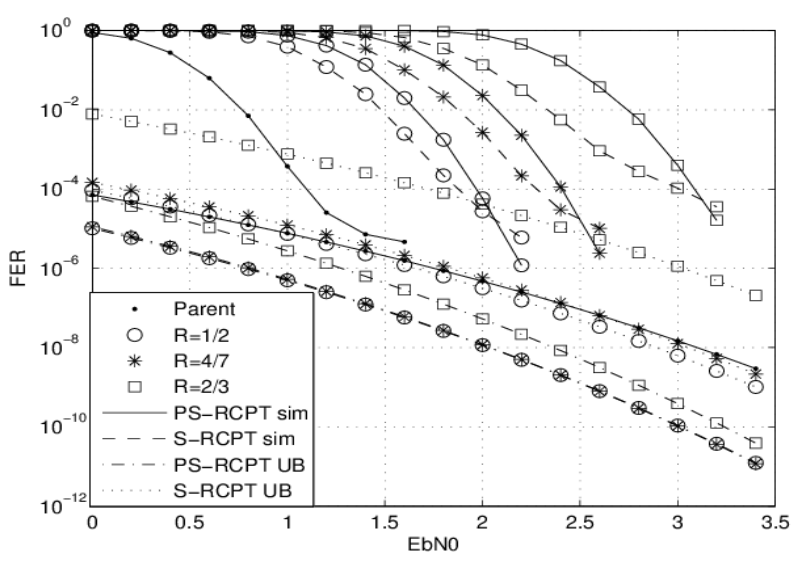

Fig. 3. FER of RCPT codes with different code rates. $k=1248$.

are illustrated in Table 3, where $L=3$. It can be readily noted that, the non-periodic puncturing patterns have favorable weight spectrum than the periodic puncturing for the same code rate as expected. As for the periodic puncturing pattern, the weight spectrum sequence with the puncturing period of $P=8$ is larger than those with the puncturing period of $P=4$, especially in higher code rate cases. This implies that the non-periodic puncturing pattern will outperform the periodic puncturing pattern, and the periodic puncturing with larger period will have better performance, as will be validated in Section IV. On the other hand, it is also observed that difference is minor when the code rate is low.

\section{NumericAl RESUlts}

In order to examine the performance of OWSS criterion based puncturing pattern design introduced in Section III, all the RCPT codes in Table 1 and Table 2 are simulated over the AWGN channel. In all simulations, the enhanced MaxLog-MAP is used and the iteration number is fixed to 8 . The frame error rate (FER) and bit error rate (BER) performance of the RCPT codes in Table 1 are illustrated in Fig. 1 and Fig. 2, respectively. It can be predicted by the union bound estimate from the weight spectrum of the $1 / 2$-code rate RCPT codes that, all PS-RCPT codes can obtain a lower error floor than

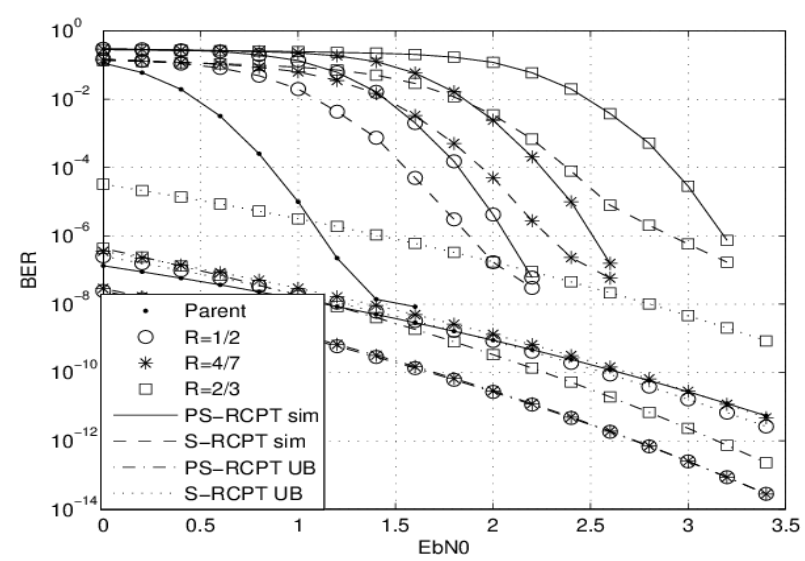

Fig. 4. BER of RCPT codes with different code rates. $k=1248$.

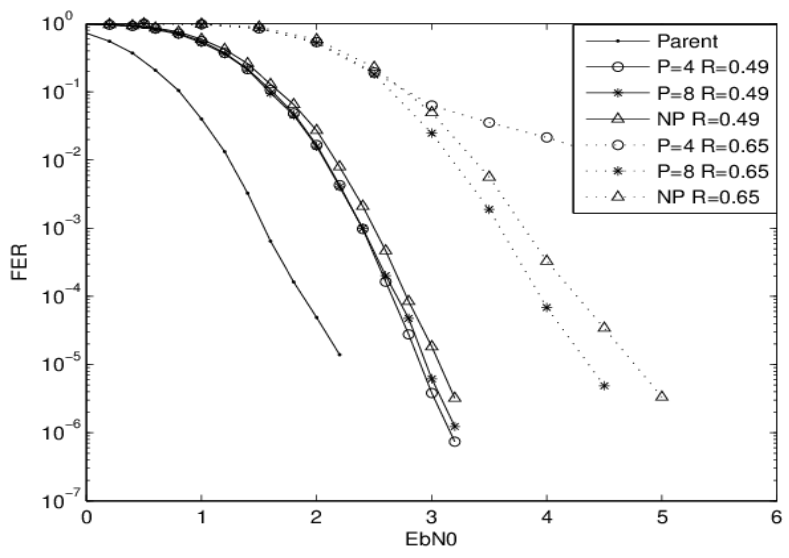

Fig. 5. FER of RCPT codes with different $P$.

the $1 / 3$-code rate mother code within the high SNR region. The PRP-RCPT code with $66 \%$ systematic bit puncturing ratio (we consider this ratio since its distance spectrum is better than the PRP-RCPT code with $57.1 \%$, as illustrated in Table 1) can achieve better error floor performance than the S-RCPT code, which complies with [8]. However, it can be observed that the performance of PRP-RCPT codes is obviously inferior to the OWSS-criterion based optimized PS-RCPT codes. On the other hand, it should be noted that, compared with the S-RCPT code, both PS-RCPT codes and PRP-RCPT codes exhibit some degradations in the waterfall region decoding performance. However, it can also be noted that the PS-RCPT codes with a small information puncturing ratio of about $25 \%$ can achieve a waterfall region performance close to that of S-RCPT codes, while achieving a much better error floor region performance. This observation explicitly suggests that a reasonable choice of the information puncturing ratio is very important for us to achieve a tradeoff between the waterfall region performance and the error floor region performance. If the RCPT codes are supposed to work in moderate SNR region and the fast decoding convergence in waterfall region is required, we should use a smaller information puncturing ratio; If the RCPT codes are supposed to work in high SNR region and the high reliability is desired, it is better to consider a larger information puncturing ratio. 


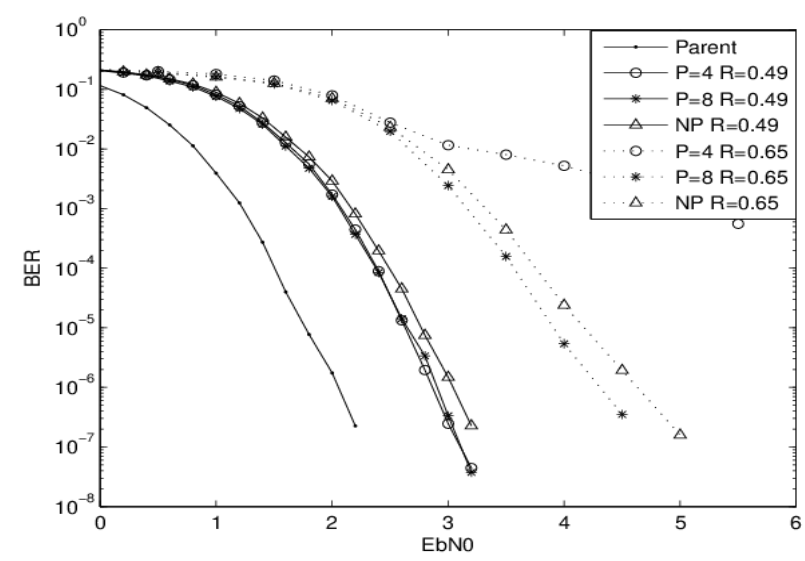

Fig. 6. BER of RCPT codes with different $P$.

The FER and BER performance of PS-RCPT codes and SRCPT codes with different code rates are presented in Fig. 3 and Fig. 4, respectively. It is shown that the S-RCPT codes always achieve a better waterfall region decoding performance than its PS-RCPT counterparts for the fixed information puncturing ratio of $50 \%$, and the performance loss in waterfall region is less than $0.5 \mathrm{~dB}$. This phenomena is owing to the punctured information bits, and the performance loss can be compensated by reasonably chosen information puncturing ratio, as we have addressed in the previous discussion. With the increase in the SNR, the relationship will be completely reversed: now the OWSS criterion based PS-RCPT codes outperform the S-RCPT codes. As for the error floor performance, it is observed that all PS-RCPT codes can achieve a lower error floor than S-RCPT codes for three code rates of $1 / 2,4 / 7$ and $2 / 3$, which comply with the weight spectrum in Table 2 . It can also be seen from Fig. 3 and Fig. 4 that, even when the code rate is increased to $2 / 3$, the OWSS criterion based PSRCPT codes can still obtain a lower error floor performance than the half rate S-RCPT codes and the original $1 / 3$ rate mother codes. Although there exists a degradation in the error floor region performance with the increase in the code rate for the OWSS criterion based PS-RCPT codes, the error floor region performance variation is comparatively small. On the other hand, the error floor region performance of the S-RCPT codes are very sensitive to the increase in the code rate. This insensitivity to the increase in the code rate makes the PS-RCPT codes an attractive adaptive Turbo coding scheme within moderate to high SNR region.

Fig. 5 and Fig. 6 illustrates the achieved FER and BER performance of periodic puncturing pattern and non-periodic puncturing pattern in Table 3 for the information size of $k=320$. We observe from simulation results that, when the punctured information ratio is high (in our analysis, up to $25 \%$ ), the PS-RCPT codes exhibit different waterfall region performance characteristics when comparing with the S-RCPT codes [6]. Firstly, the increase in the puncturing periods will not definitely give rise to the improved performance; Secondly, non-periodic puncturing will not always give rise to a better waterfall region performance. Both differences can be explicated by the degradation owing to the removal of some in- formation bits, especially when the punctured information ratio is high. This observations comply with the previous results, namely, the punctured information ratio should be carefully devised to guarantee the achieved performance tradeoff between the waterfall region and error floor region. Secondly, the results in Fig. 5 and Fig. 6 also remind us that, we should try to include the waterfall region performance estimation metric in the PS-RCPT codes to effectively satisfy the waterfall region performance requirements in adaptive Turbo coding design in practical applications. That will be the future direction for improved adaptive Turbo coding design work.

\section{CONCLuSions}

In this paper, the optimal puncturing pattern design for adaptive Turbo coding scheme is investigated for the LTE standard Turbo codes. Through employing the constrained subcode algorithm to calculate the low weight spectrum sequence, the modified OWSS criterion is utilized to determine the optimal puncturing pattern. Two algorithms are proposed to determine the optimal periodic puncturing pattern and nonperiodic puncturing pattern for PS-RCPT codes. It has been shown that the optimized PS-RCPT codes can achieve better error floor performance than S-RCPT codes at the cost of some degradation in the decoding convergence within waterfall region. Meanwhile, the OWSS-criterion based PS-RCPT codes are superior to the pseudo-random puncturing (PRP) based PS-RCPT codes. In addition, there exists an appropriate information bit puncturing ratio to generate PS-RCPT codes with reasonable tradeoff in the waterfall region and the error floor region performance. The OWSS based puncturing pattern design provides an effective method to generate adaptive Turbo coding scheme to satisfy the performance required by applications.

\section{REFERENCES}

[1] J. Hagenauer, "Rate-compatible punctured convolutional codes (RCPC codes) and their applications," IEEE Trans. Commun., Vol. 36, No. 4, pp. 389-400, Apr. 1998.

[2] A. S. Barbulescu and S. S. Pietrobon, "Rate compatible turbo codes," Electronics Letters, Vol. 31, No. 7, pp. 535-536, March 1995.

[3] D.N.Rowitch, L.B.Milstein, "On the performance of hybrid FEC/ARQ systems using rate compatible punctured turbo(RCPT) codes," IEEE Trans. Commun., Vol. 48, No. 6, pp. 948-959, June 1999.

[4] F. Babich., G. Montorsi, and F. Vatta, "Some notes on rate-comptaible punctured Turbo codes (RCPTC) design," IEEE Trans. Commun., Vol. 52, No. 5, pp. 681-684, May 2004.

[5] E. Rosnes, O. Ytrehus, "On the construction of good families of ratecompatible punctured turbo codes," in Proc. Of IEEE ISIT2005, pp. 602 606, 2005.

[6] Jiaxiang Li, Qingchun chen, Suyue Gao, Zheng Ma, Pingzhi Fan, "The Optimal Puncturing Pattern Design for Rate-Compatible Punctured Turbo Codes," in Proc. of WCSP'09, pp. 1-5, 2009.

[7] R. Garello, P. Pierleoni, and S. Benedetto, "Computing the free distance of turbo codes and serially concatenated codes with interleavers: Algorithms and applications," IEEE Journal on Selected Areas in Communications, Vol. 19, No. 5, pp. 800-812, May 2001.

[8] I. Chatzigeorgiou, M. R. D. Rodrigues, I. J. Wassell, R. A. Carrasco, "Analysis and design of punctured rate-1/2 turbo codes exhibiting low error floors," IEEE Trans. Commun., Vol. 27, No. 6, pp. 944-953, 2009.

[9] 3rd Generation Partnership Project, "Evolved Universal Terrestrial Radio Access (E-UTRA): Multiplexing and Channel Coding", 3GPP TS 36.212 v8.6.0, March 2009.

[10] Qingchun Chen, Jiaxiang Li, Pingzhi Fan, Vahid Tarokh, ”An Exponential Model for Evaluating Error Performance of Turbo codes," in Proc. of IEEE Globecom 2010, Miami, FL, 2010. 